\title{
Mechanical properties at high strain-rate of lead core and brass jacket of a NATO $7.62 \mathrm{~mm}$ ball bullet
}

\author{
L. Peroni ${ }^{1}$, M. Scapin ${ }^{1}$, C. Fichera ${ }^{1}$, A. Manes ${ }^{2}$, and M. Giglio ${ }^{2}$ \\ ${ }^{1}$ Politecnico di Torino, Department of Mechanical and Aerospace Engineering (DIMEAS), \\ Corso Duca degli Abruzzi 24, 10129 Turin, Italy \\ 2 Politecnico di Milano, Department of Mechanical Engineering, via la Masa 1, 20156 Milano, Italy
}

\begin{abstract}
Numerical simulations are now an actual option in order to try to reproduce and understand the mechanical response in components subjected to extreme loading conditions, like in a ballistic impact. A correct materials calibration is therefore necessary in order to extract the materials parameters. In this work the simple and widely used Johnson-Cook model was used to analyse the experimental data obtained for the characterization of the bullet materials. The bullet under investigation is a full metal jacket ball, with a lead-antimony alloy core and a brass jacket. The experimental tests cover a wide range in strain-rate, starting from quasi-static tests up to high dynamic tests performed on a standard Split Hopkinson Pressure Bar setup. In general, there is a great lack in strain-rate sensitivity and failure data. Pure lead is very soft and ductile, so antimony is used to give greater hardness and strength. The results of this study show a significant strain-rate influence for this alloy that can be associated with the presence of the lead-antimony phases and their structures. Also in case of the brass the results showed significant strain-rate sensitivity in the material response.
\end{abstract}

\section{Introduction}

The main objective of this work is the mechanical characterization of the materials involved in high strain-rate scenario, like ballistic impact. Ballistic impact implies that the materials (both of the target and the bullet) are subjected to very high strain-rate combined with high levels of deformation, temperature and, of course, damage, especially in the zone very near to the impact.

The case study analysed is the bullet impact on the helicopter tail rotor transmission shaft [1]. The shaft is realized in Al6061-T6 alloy and the bullet is a common $7.62 \times 51 \mathrm{~mm}$ Ball full metal jacket, with the core in leadantinomy alloy and the jacket in brass. Since the final goal will be the numerical simulation of this phenomenon, the parameters identification, based on an analytical model, is therefore necessary. In particular, in this work the attention is focused on the mechanical characterization and parameters identification for the bullet materials.

In a previous work [2] Gilioli et al. performed the material calibration for the aluminium alloy of the shaft in the static regime with the Johnson-Cook (J-C) model [3]. Moreover, Gilioli et al. focused the attention on the identification of the failure model. The failure model used was the ductile criterion of Bao-Wierzibicki, which expresses the strain at failure in function of the level of triaxiality. This model is suitable in case of complex loading conditions, in which usually the level of triaxiality varies in a wide range being difficult to predict a priori. The model was calibrated and the results were compared with those obtained with the standard J-C failure model. Instead, both for the strain-rate sensitivity of the shaft and the bullet materials behaviours, reference parameters from the scientific literature were used $[4,5]$. The problem in this sense is that, in particular for lead-antimony alloy, there is a great lack in strain-rate sensitivity and failure data. Some results on the high strain-rate influence on the mechanical behaviour (i.e. [6]) can be found but mainly for pure lead. For what concerns the brass used to make cartridges, the $\mathrm{J}-\mathrm{C}$ parameters can be found in [3].

The work, proposed by Manes et al. [7], improved the work [2]. The aluminium alloy was characterized performing a numerical inverse optimization in order to extract, from both quasi-static and high strain-rate compression tests, the strain-hardening and strain-rate coefficients of the J-C model.

This work has the aim to further improve the materials characterization, focusing the attention on the bullet. A campaign of experimental tests is performed starting from quasi-static up to very high strain-rate loading conditions, obtained with a standard Split Hopkinson Pressure Bar setup. From the experimental results the strainhardening parameters and the strain-rate sensitivity are obtained. Otherwise, the model calibration in function of the temperature is not performed. The temperature influence is considered with a simplified approach: the authors assumed the temperature parameters from [3] for the brass and a linear decrease of the mechanical strength with the temperature increase for the lead alloy.

\section{Bullet description}

In recent years, the importance to understand the actual behaviour of components subjected to ballistic impacts leads to directly use military bullet in the research fields.

The bullet under analysis in this work is the $7.62 \times$ $51 \mathrm{~mm}$ Ball. It is a full metal jacket bullet with the core in lead-antimony alloy and the jacket in brass. This type of bullet has a limited penetrating power, but thanks to the ductility of the core, it is heavy deformed during the impact against a metallic target. In this way it invests a greater surface, causing high damage. 


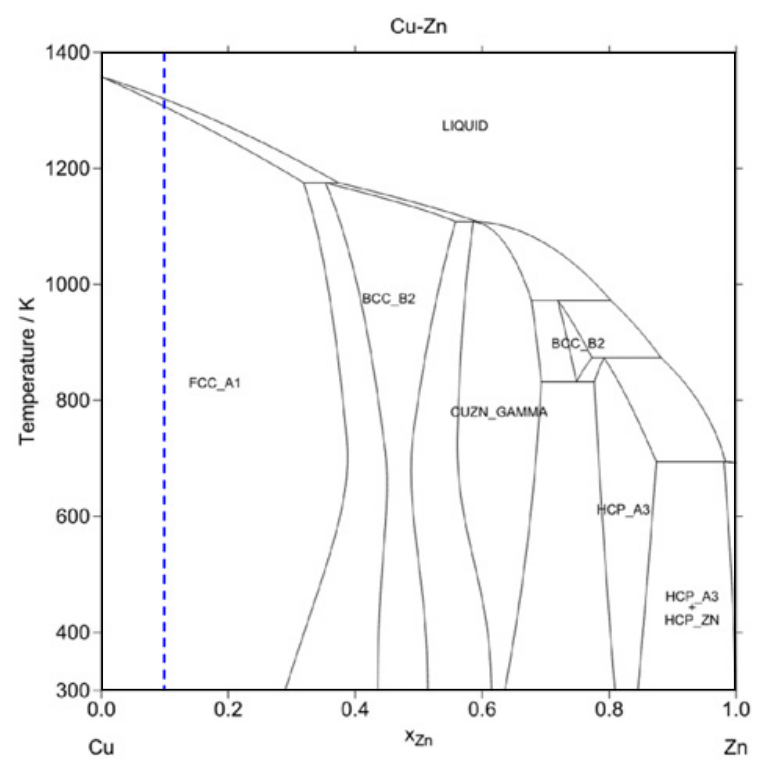

Fig. 1. Phase diagram of $\mathrm{Cu}-\mathrm{Zn}$ alloys (source MTDATA database). The dashed line refers to the alloy analysed.

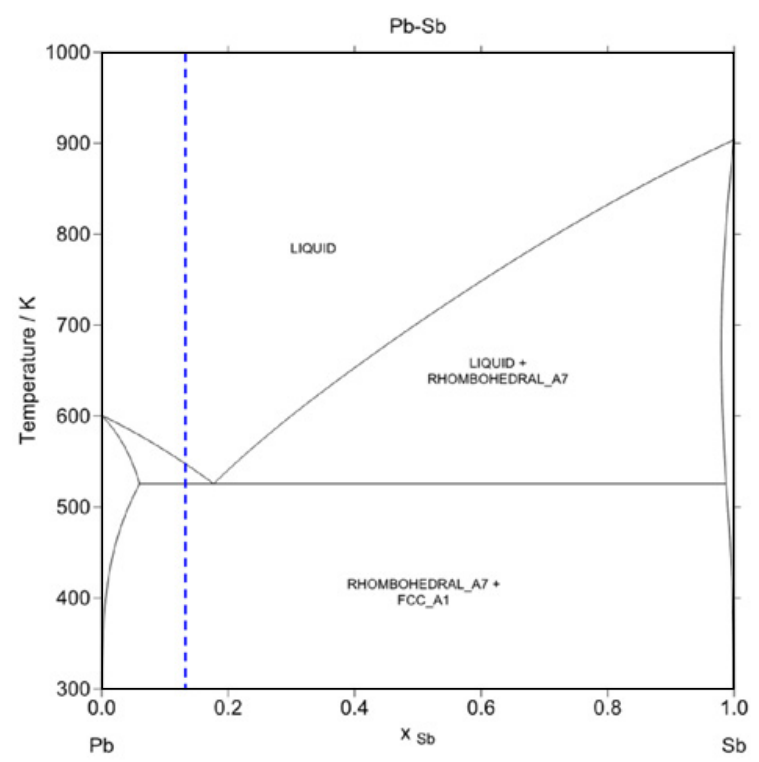

Fig. 2. Phase diagram of Pb-Sb alloys (source MTDATA database). The dashed line refers to the alloy analysed.

The chemical compositions of the bullet alloys are: 86 wt.- $\% \mathrm{~Pb}$ and 14 wt.- $\% \mathrm{Sb}$ for the core and $90 \mathrm{wt} . \% \mathrm{Cu}$ and 10 wt.- $\% \mathrm{Zn}$ for the jacket. These types of alloys are commonly used in the bullet construction as reported by Borvik et al. in [8].

Pure lead is very soft and ductile, so antimony is generally used to give greater hardness and strength. The contents of antimony generally used in this application can range from 0.5 to $25 \%$.

In figures 1 and 2 the phase diagrams of the two alloys are reported. In correspondence to a weight percentage of zinc equal to $10 \mathrm{wt} .-\%$, as in the case analyzed, the diagram of the brass shows the presence of one phase at the solid state. This phase is characterized by a FCC structure which remains very similar to that of the pure copper. This is

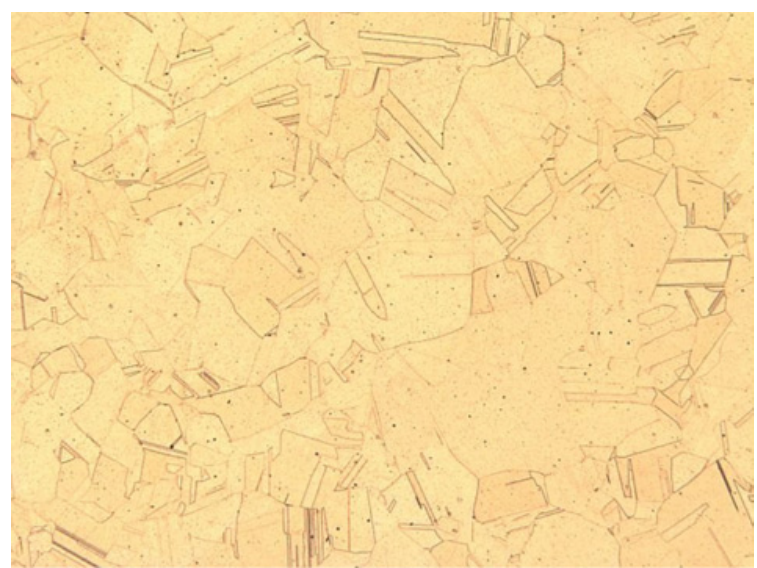

Fig. 3. Example of micrograph of the brass: for low percentage of zinc the two materials are soluble at the solid state and create the single phase FCC_A1.

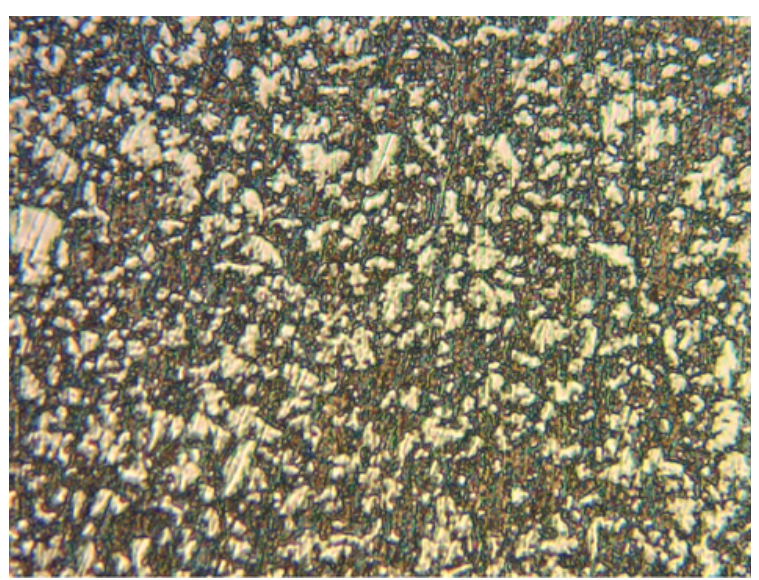

Fig. 4. Micrograph of the lead-antimony alloy used in this work, in which it is possible to appreciate the two phases at the solid state: the dark part is the FCC_A1 structure of the $\mathrm{Pb}$ and the light part is the rhomboedral_A7 structure of the Sb.

also confirmed by looking the micrograph of the material, as shown in figure 3. The thermal properties are very similar to those of pure copper. The melting temperature is assumed from the diagram of figure 1 equal to $1300 \mathrm{~K}$ and the specific heat at constant pressure equal to $385 \mathrm{~J} / \mathrm{kg} / \mathrm{K}$. The lead-antimony alloy binary phase diagram (figure 2) shows a eutectic point. At the $14 \mathrm{wt}$.\% of $\mathrm{Sb}$, there is the presence of two phases at the solid state: the FCC_A1 structure of the $\mathrm{Pb}$ and the rhomboedral_A7 structure of the $\mathrm{Sb}$. This is also confirmed by the micrograph reported in figure 4. The melting temperature is assumed from the diagram of figure 2 equal to $525 \mathrm{~K}$. The specific heat at constant pressure is assumed equal to $140 \mathrm{~J} / \mathrm{kg} / \mathrm{K}$ (average weighted value between the lead and the antimony heat capacities).

For the material characterization, the idea is to carry out experimental tests taking into account as much as possible the residual stress on the material due to the production process of the bullet.

The specimens of the lead-antimony alloy are obtained directly from the cylindrical part of the bullets core. 

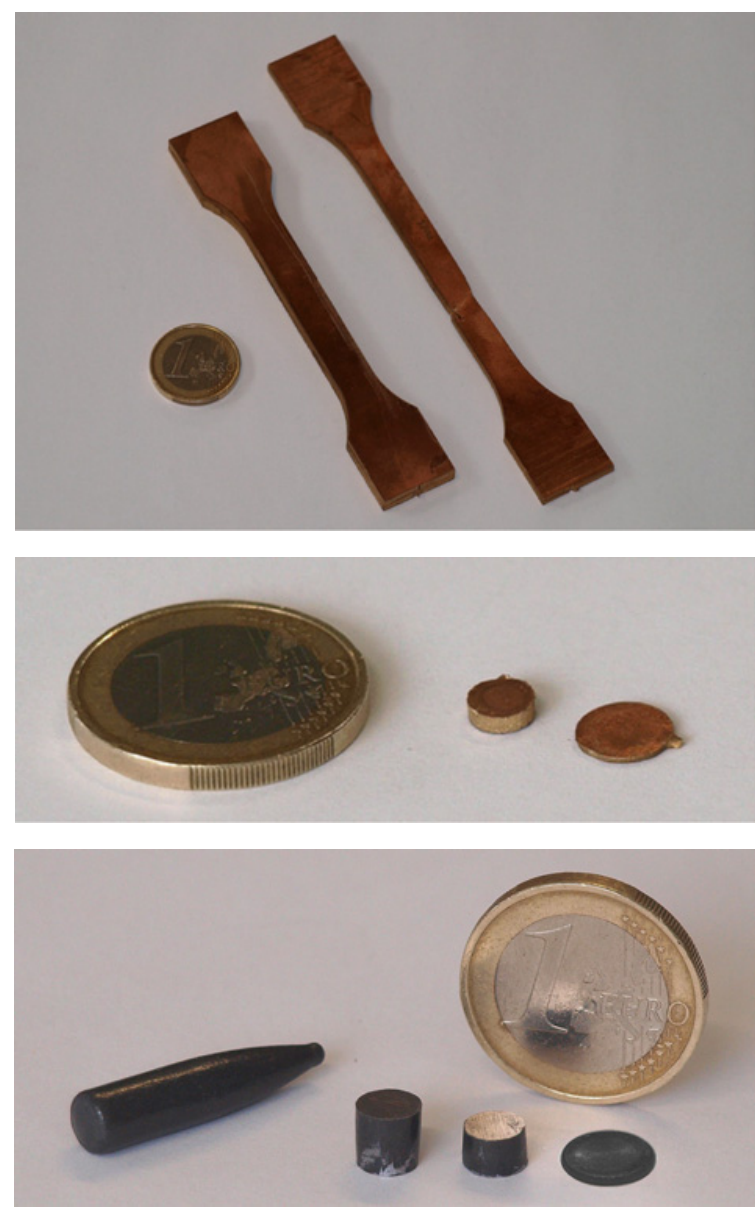

Fig. 5. Specimens used in the experimental tests (before and after the test): (top) tensile specimen for brass (double layer); (middle) compression specimen for brass (single layer); (bottom) compression specimens for lead-antimony alloy.

For this reason, the experimental tests performed on this material are compression tests. The specimens length considered in the analysis varies between 4 and $6 \mathrm{~mm}$ with an average diameter of $6 \mathrm{~mm}$.

For what concerns the brass, it is not possible to obtain specimens directly from the jacket. For this reason, the specimens are obtained from the metal sheets used for the bullet construction with a waterjet cutting process. The cylindrical specimens obtained in this way are very thin and small and they could be affected by the inhomogeneity of the sheet and for this reason not be representative of the global response of the material. Then also tensile specimens are obtained in order to compare the results in terms of quasi-static material response obtained with the two geometries. The specimens used for the compression tests have an average diameter of $5.1 \mathrm{~mm}$ and an average length of $1.5 \mathrm{~mm}$. For the compression tests two types of specimens are used: single layer specimens and double layer ones, in which two specimens are jointed. The tensile specimens are obtained with a cross section of about $10 \mathrm{~mm} \times 3 \mathrm{~mm}$ (double layer) and a gauge length of $65 \mathrm{~mm}$.

In figures 5 the pictures of the specimens used for the model calibration are shown.

\section{Material model}

It is important to remark that a material characterization must count on a specified analytical model from which the number of strength parameters and the types of experimental tests to be performed depend. For this reason, it is very important that experimental tests and numerical modelling go hand in hand in order to avoid both an inadequate and an overflowing number of data. So, first of all it is necessary to choose the material model on which it is consequently possible to plan the experimental tests.

In this work the analytical model chosen for the study is the Johnson-Cook (J-C) [3] model. It is one of the simplest models able to predict the mechanical behaviour of the materials under different loading conditions. Besides, it is one of the most used models, so it is implemented in many FEM codes and it is quite easy to find in literature the values of J-C parameters for different materials. Several authors used the $\mathrm{J}-\mathrm{C}$ model, or its modified formulations, in order to investigate and describe problems in which the strain-rate component is relevant, and in particular in case of ballistic impacts i.e. [9].

The J-C formulation expresses the flow stress as the product of three terms, which respectively, describe the strain hardening, strain-rate and temperature influences. The flow stress is defined as follows:

$$
\sigma_{y}=\left(A+B \varepsilon_{\mathrm{pl}}^{n}\right)\left(1+C \ln \frac{\dot{\varepsilon}_{\mathrm{pl}}}{\dot{\varepsilon}_{0}}\right)\left(1-\left(\frac{T-T_{\mathrm{r}}}{T_{\mathrm{m}}-T_{\mathrm{r}}}\right)^{m}\right)
$$

in which $A$ is the elastic limit strength, $B$ and $n$ are the work hardening parameters, $C$ is the strain-rate sensitivity coefficient and $m$ describes the thermal softening. In the LS-DYNA formulation [10], $\dot{\varepsilon}_{0}$ represents the quasi-static strain-rate threshold, which identifies the highest strainrate for which the strain-rate effects on the flow stress are negligible. The $\mathrm{J}-\mathrm{C}$ model is a multiplicative model: this implies that the effects of plastic strain, strain-rate and temperature are assumed to act independently on the flow stress evolution. In this case, a strain-rate or temperature variation implies only a scaling and not a modification in the shape of the strain hardening curve.

\section{Experimental tests}

According to the fact that the $\mathrm{J}-\mathrm{C}$ material model is uncoupled in plastic strain, strain-rate and temperature effects, the experimental tests are managed exchanging one parameter at a time. This greatly simplifies the experimental phase. As a matter of fact, for the identification of the strain hardening curve, experimental tests at room temperature and quasi-static rate should be sufficient. The identification of temperature could be performed on the basis of experimental tests at different temperatures in quasi-static conditions. Finally, the strain-rate sensitivity could be identified from tests performed varying the strain-rate at room temperature. It is important to remark that it can be a quite simple approach. The phenomenon under analysis is strongly thermo-structural coupled: the structural-mechanics material conditions produce the rise in temperature that consequently modifies the mechanical 
material response. Usually, due to the rise in temperature, there is a modification (reduction) in the effect of the strain-rate on the flow stress. Moreover, the thermal softening has to be taken into account. It is essentially due to heat conversion of mechanical work. The thermal softening produces significant effects on the material strength especially in case of high strain-rate conditions, since the phenomenon evolution is adiabatic, and where strain is localized.

In this work the identification of strain-hardening and strain-rate parameters to introduce in the numerical model is the final objective. The temperature influence is considered with a simplified approach: the authors assumed the temperature parameter $(m=1.68)$ from [3] for brass and a linear decrease $(m=1)$ between the reference temperature $\left(T_{\mathrm{r}}\right)$ and the melting temperature $\left(T_{\mathrm{m}}\right)$ of the mechanical strength with the temperature increase for lead alloy.

For each testing condition at least three repetitions are carried out. The experimental tests cover a range in strain-rate between $1 \times 10^{-2} \mathrm{~s}^{-1}$ up to $5 \times 10^{3} \mathrm{~s}^{-1}$. The quasi-static loading conditions are obtained via general purpose electro-mechanical testing machine (Zwick Z-100). High strain-rate tests are carried out with a standard Split Hopkinson Pressure Bar (SHPB) setup. The bars (striker, input and output bars) are made in high strength (martensitic) stainless steel (17-4 PH) and have a diameters of $10 \mathrm{~mm}$. The striker length varies between 1000 and $400 \mathrm{~mm}$ in order to increase the maximum achievable strain-rate. Both input and output bars are $3 \mathrm{~m}$ long. The strain signals in the bars are measured by a half bridge of semiconductor strain gages (KYOWA KSP-1-350-E) and recorded with a National Instruments PC-6133 data acquisition board (14 bit, $3 \mathrm{MHz}$ ).

\section{Results and discussion}

In figure 6(a) and 6(b) experimental results in terms of true stress-true plastic strain are shown, respectively for brass and lead-antimony alloy. The curves are obtained analytically under the hypothesis of volume conservation in the plastic domain. For sake of clarity, for both the materials, only two curves at different strain-rates are reported. In case of brass, the quasi-static results shown in the figure are which obtained from tensile tests. The curves reported reach different values of plastic strain. This can be due to different reasons. The tensile curves are limited by the specimen failure. The length of high strain-rate curves depends on the length of the compressive pulse produced on the SHPB setup, which depends on the striker length.

Looking qualitatively the results, it is possible to conclude that, for both the materials, the results show the influence of the strain-rate on the material response. As a matter of fact, it is possible to clearly identify the different levels of strain-rate given that they correspond to significant increases in terms of strength of the materials.

The results of the compression tests performed on the brass are quite scattered. This should be due to the fact that the specimens are very small and thin. Moreover, they come from a large sheet, which probably is not homogenous in terms of residual stresses. This is
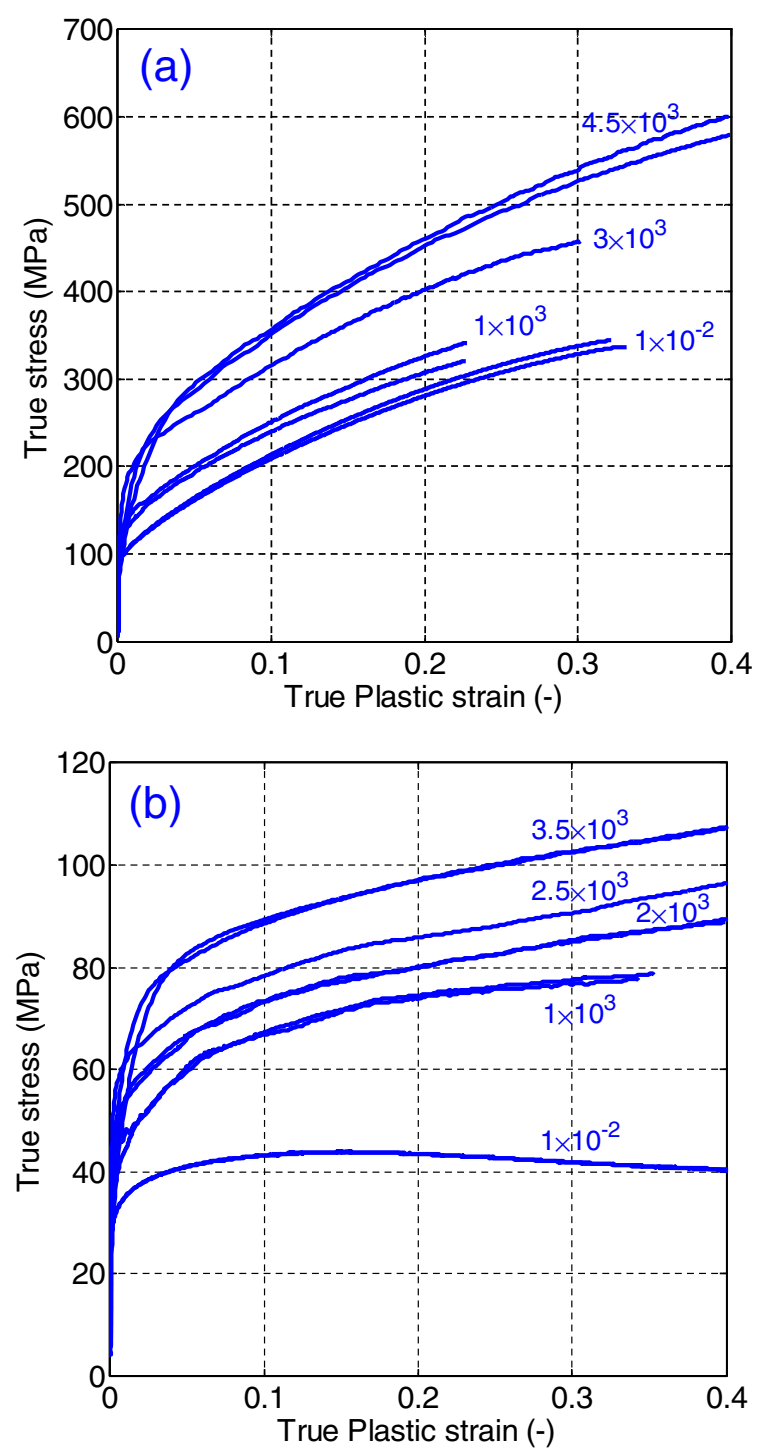

Fig. 6. Experimental results in terms of true stress-true plastic strain at four different strain-rates $\left(\mathrm{s}^{-1}\right)$ for brass (a) and leadantimony alloy (b).

confirmed by the result obtained in tension. In this case the results show a good repeatability: the specimen is big enough to average the inhomogeneity. The brass shows a significant hardening phase and the plastic strain at failure can be estimated over $30 \%$. The increment in the material strength due to the increase in the strain-rate is significant in the range of strain-rate investigated $\left(10^{3} \div 10^{4} \mathrm{~s}^{-1}\right)$. The brass considered in this work has a FCC structure, which corresponds to low strain-rate sensitivity in low strainrate regime (until $10^{3} \mathrm{~s}^{-1},[3]$ ): this aspect will be further investigated.

Also for the lead-antimony alloy, until $10 \mathrm{~s}^{-1}$ relative small strain-rate sensitivity was expected in accordance to the results available in case of pure lead [6]. The results obtained in this work in the range over $10^{3} \mathrm{~s}^{-1}$ show quite significant strain-rate sensitivity. This could be associated with the presence of the lead-antimony phases and their structures. For this alloy the scattering of the results is more contained respect to the brass. For lead-antimony 
alloy it is not possible to estimate a value for the strain at failure since only compression tests are performed. From the available results, it is possible to assess that the material is quite ductile. Besides, from the experimental results reported in figure 6(b), it appears evident the different shape between the quasi-static and dynamic curves. This is probably due to a damage mechanism of the brittle phase (antimony) which produces more effects at low rate of deformation.

\subsection{Material model identification}

In this paragraph the procedure used for extracting the material model parameters is described. The analysis of the experimental data is performed with MATLAB. The use of an optimization tool could allow the implementation of a multi-objective procedure, in which all the parameters are obtained on the basis of both static and dynamic curves. The problem encountered with the J-C model is that the variation of some parameters produces the same effect on the flow stress of the variation of other parameters [11]. For this reason, the authors choose to implement a stepby-step procedure in order to control the optimized values and check their verisimilitude.

The first step is a single objective optimization, based on the minimization of the mean squared error, in which for each experimental curve $A_{1}, B_{1}$ and $n_{1}$ are obtained on the basis of the equation (2):

$$
\sigma_{y}=\left(A_{1}+B_{1} \varepsilon_{\mathrm{pl}}^{n_{1}}\right)\left(1-\left(\frac{T-T_{\mathrm{r}}}{T_{\mathrm{m}}-T_{\mathrm{r}}}\right)^{m}\right)
$$

The temperature evaluation is performed under the isothermal assumption in case of quasi-static tests and under the adiabatic assumption in case of dynamic tests.

In the second step, the value of the hardening exponent is fixed to the average of those obtained during the first step. This allows taking into account the shape of all the curves (and not only that of the static curves) for the determination of the $\mathrm{J}-\mathrm{C}$ reference hardening curve. Then a second single objective optimization is performed for each curve fixing also the ratio $\left(r_{1}\right)$ between $A_{1}$ and $B_{1}$ equal to the average value of those obtained from the first step. In this step only $B_{2}$ is optimized, according to the equation (3):

$$
\sigma_{y}=\left(B_{2} \bar{r}_{1}+B_{2} \varepsilon_{\mathrm{pl}}^{\bar{n}_{1}}\right)\left(1-\left(\frac{T-T_{\mathrm{r}}}{T_{\mathrm{m}}-T_{\mathrm{r}}}\right)^{m}\right)
$$

In this way the strain-rate influence is condensed in the strain-hardening coefficients $B_{2}$. For what concerns the strain-rate sensitivity, usually, only the parameter $C$ of the second term of the J-C model (Eq. (1)) is considered as an optimization variable, while $\dot{\varepsilon}_{0}$ is set equal to $1 \mathrm{~s}^{-1}$. In some FE codes (i.e. LS-DYNA, Abaqus) this choice would mean that the strain-rate influence is neglected for all the experimental set data with strain-rate less than this value. If also $\dot{\varepsilon}_{0}$ is used as optimization variable, an improvement in the experimental data fit could be obtained [11]. The set of strain-hardening coefficients is normalized with respect to the static mean value $\left(\sigma / \sigma_{\text {st }}\right)$ and reported in function

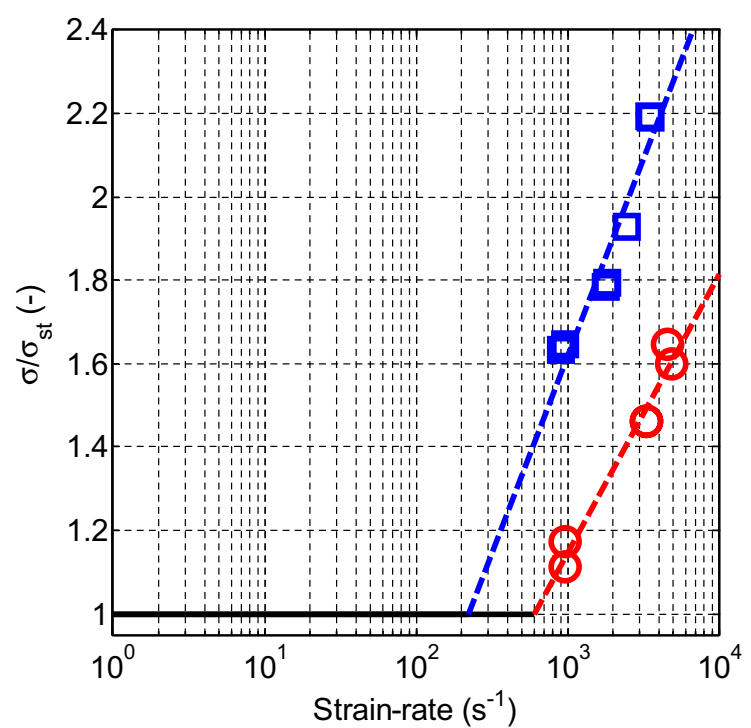

Fig. 7. Strain-rate sensitivity parameters identification. Each experimental curve is represented (markers: squared for leadantimony alloy, circled for brass) by the ratio between its strainhardening coefficient $B_{2}$ and the mean of the static values (obtained for a strain-rate of about $10^{-2} \mathrm{~s}^{-1}$ ). The optimized $\mathrm{J}-\mathrm{C}$ models are also shown (lines) for the two materials. The diagram is bounded below to a strain-rate value of $1 \mathrm{~s}^{-1}$, which is the standard value of $\dot{\varepsilon}_{0}$ in the J-C model: using this value, for both the materials, a worst interpolation would be obtained.

of the strain-rate in a semi-log plane in figure 7. For both the materials, the experimental data distribution in the stress vs. strain-rate plane justifies the choice of using the J-C model. As a matter of fact, the results can be well approximated with a piecewise linear function. The parameter $C$ is obtained interpolating the dynamic results with a linear function. Since the final objective of this study will be the modelling of a ballistic impact, the J-C calibration is focused in the strain-rate regime between $10^{3}$ and $10^{4} \mathrm{~s}^{-1}$. Unfortunately for higher values of strain-rate, at the moment, there are not experimental data available. Probably, in the ballistic impact strain-rate over $10^{4} \mathrm{~s}^{-1}$ should be achieved: in this case the use of the obtained $\mathrm{J}-\mathrm{C}$ model is an approximate extrapolation.

The parameter $\dot{\varepsilon}_{0}$ is extracted as intersection between the horizontal line $\left(\sigma / \sigma_{\text {st }}=1\right)$ passing through the static results and the line with slope equal to $C$ get from the dynamic results. In figure 7 the diagram is bounded below in strain-rate to $1 \mathrm{~s}^{-1}$ : this value is the standard value used in the J-C formulation. From the diagram the improvement obtained optimizing also $\dot{\varepsilon}_{0}$ appears evident.

The results for both the materials are summarized in Table 1.

\section{Conclusions}

In this work the Johnson-Cook material model calibration was performed. The objective was the characterization of the materials of the $7.62 \times 51 \mathrm{~mm}$ Ball bullet, in order to obtain the materials coefficients to introduce in numerical models of the ballistic impact against a helicopter shaft. The bullet considered is a full metal jacket one with 
Table 1. J-C parameters.

\begin{tabular}{|c|c|c|c|c|c|}
\hline Material & $A(\mathrm{MPa})$ & $B(\mathrm{MPa})$ & $n(-)$ & $C(-)$ & $\dot{\varepsilon}_{0}\left(\mathrm{~s}^{-1}\right)$ \\
\hline Brass & 80 & 500 & 0.605 & 0.290 & 604 \\
\hline Lead- & & & & & \\
\hline antimony & 1 & 61 & 0.163 & 0.410 & 221 \\
\hline alloy & & & & & \\
\hline
\end{tabular}

the core in lead-antimony alloy and the jacket in brass. The experimental tests performed covered a wide range in strain-rate, starting from quasi-static tests up to high strain-rate tests carried out on a standard Split Hopkinson Pressure Bar setup.

For the material characterization, the specimens were obtained directly from the bullet in case of lead-antimony alloy and from the sheet used for the jacket in case of brass. This is in order to take into account as much as possible the residual stress on the material due to the production process of the bullet.

The brass shows a significant hardening phase and the plastic strain at failure was estimated over $30 \%$. The strain-rate variation produces a scaling between the curves and the strain-rate sensitivity is quite significant.

The lead-antimony alloy is quite ductile. For this material it was not possible to estimate a value for the strain at failure since only compression tests were performed. In literature there is a great leak of results for this alloy, especially at high strain-rate. The results obtained showed significant strain-rate sensitivity in the range covered with the SHPB tests. The shape of the stress-strain curves appeared very different in quasi-static and dynamic regime. This is probably due to the damage mechanism of the brittle phase (antimony) which produces more effects at low rate of deformation.

\section{References}

1. L. Giudici, A. Manes, M. Giglio, $25^{\text {th }}$ International Symposium on Ballistic pp:1058-1065 (2010)

2. A. Giglioli, A. Manes, M. Giglio, Calibration of a constitutive material model for AL-6061-T6 alumininum alloy, Proceedings of $5^{\text {th }}$ International Conference on Advanced Computational Engineering and Experimentig (2010)

3. G.R. Johnson, W.A. Cook, A constitutive model and data for metal subjected to large strains, high strain rates and high temperatures, Proocedings of 7 th International Symposium on Ballistic, pagg :541-547 (1983)

4. D.R. Leuser, G.J. Kay, M.M. Le Blanc, Modeling Large-Strain, High-Rate Deformation in Metals, $3^{\text {th }}$ Biennial Tri. Lab. Eng. Conf. Modeling and Simulation (1999)

5. ASM Handbook Volume 02 - Properties and Selection: Nonferrous Alloys and Special-Purpose Materials, (1998)

6. U.S. Lindhom, J. Mech. Phys. Solids, 12 pp: 317335

7. A. Manes, L. Peroni, M. Scapin, M. Giglio, Procedia Engineering, 10 pp:3477-3482 (2011)

8. T. Børvik, S. Dey, A.H. Clausen, Int. J. Imp. Eng., 36 (2009)

9. M. Buyuk, C-D.S. Kan, N.E. Bedewi, A. Durmus, S. Ulku, Moving Beyond the Finite Element, a Comparison Between the Finite Element Methods and Meshless Methods for a Ballistic Impact Simulation, $8^{\text {th }}$ International LS-DYNA Users Conference (2004)

10. B. Gladman, LS-DYNA® Keywords user's manual, Vol.1 - Version 971, LSTC (2007)

11. M. Scapin, L. Peroni, M. Peroni, Int. J. Imp. Eng. 40-41 pp:58-67 (2012) 